\title{
An Optimal-Transport-Based Reinforcement Learning Approach for Computation Offloading
}

\author{
Zhuo $\mathrm{Li}^{1,2}, \mathrm{Xu}$ Zhou ${ }^{1}$, Taixin $\mathrm{Li}^{1}$, Yang $\mathrm{Liu}^{3}$ \\ ${ }^{1}$ Computer Network Information Center, Chinese Academy of Sciences, Beijing 100190, China \\ ${ }^{2}$ University of Chinese Academy of Sciences, Beijing 100049, China \\ ${ }^{3}$ School of Mathematical Sciences, Beijing Normal University, Beijing 100875, China \\ \{lizhuo, zhouxu, txli\}@cnic.cn, liu.yang@mail.bnu.edu.cn
}

\begin{abstract}
With the mass deployment of computing-intensive applications and delay-sensitive applications on end devices, only adequate computing resources can meet differentiated services' delay requirements. By offloading tasks to cloud servers or edge servers, computation offloading can alleviate computing and storage limitations and reduce delay and energy consumption. However, few of the existing offloading schemes take into consideration the cloud-edge collaboration and the constraint of energy consumption and task dependency. This paper builds a collaborative computation offloading model in cloud and edge computing and formulates a multi-objective optimization problem. Constructed by fusing optimal transport and PolicyBased RL, we propose an Optimal-Transport-Based RL approach to resolve the offloading problem and make the optimal offloading decision for minimizing the overall cost of delay and energy consumption. Simulation results show that the proposed approach can effectively reduce the cost and significantly outperforms existing optimization solutions.
\end{abstract}

Index Terms - computation offloading, reinforcement learning,optimal transport, cloud computing, edge computing

\section{INTRODUCTION}

With the further development of computing power's marginalization, the traffic from the end devices (EDs) has increased dramatically. However, due to the limited resources and computing performance, the EDs may face insufficient capabilities when processing computation-intensive and timesensitive applications. To solve the problem mentioned above, computation offloading allocates tasks with a large amount of computing requirements to a server with sufficient computing resources to process and retrieve the results. Computation offloading in cloud computing transfers tasks to remote cloud servers for execution and uses its computing and storage resources to alleviate computing and storage limitations and extend the EDs battery life [1], while offloading tasks to cloud servers face problems such as unpredictable delays and long transmission distances [2]. Unlike cloud computing, edge computing that uses servers to process and analyze data has emerged and is complementary to traditional cloud computing [3], which provides cloud computing functions at the edge of the wireless access network near mobile users, providing computing services for EDs more quickly and efficiently while alleviating the pressure on the core network [4].

From the perspective of computing, offloading can significantly shorten the tasks' execution delay and reduce energy consumption in local computing. In contrast, from the perspective of communication, the upload of data and feedback of the results introduce additional time and energy consumption [5]. To solve the deficiencies of EDs in resource storage, computing performance, and energy efficiency, offloading the task of EDs to cloud servers and edge servers has become the mainstream direction of computation offloading. As the dependencies between tasks become more complex, it is more general to abstract them as Directed Acyclic Graph (DAG). On the premise of not violating mutual dependence, tasks in DAG applications can be executed in parallel in cloud servers and edge servers [6].

Considering different application scenarios and requirements, researchers have researched the decision-making problem of offloading. From the perspective of minimizing delay or energy consumption, many existing solutions propose corresponding models for offloading based on mathematical models, and solve NP-hard problems based on heuristic or approximate algorithms [7], [8]. However, these solutions rely heavily on accurate mathematical models. Therefore, it is difficult for one specific algorithm to fully adapt to the dynamic scenarios from the increasing complexity of applications.If the dynamic arrival of tasks, random changes in task attributes, user movement, and dynamic changes of wireless channels are not considered, the offloading decision process can be abstracted as a Markov Decision Process (MDP) and requires a State Transition Probability Matrix (STPM) to describe the system state's change probability under different offloading decisions [9]. After that, the optimal offloading decision can be obtained through value iteration or policy iteration.

As the STPM is difficult to obtain, the optimal policy can be learned by using Reinforcement Learning (RL) methods through continuous interaction. RL is a branch of machine learning used to learn the optimal policy when there is no information about the surrounding environment [10]-[12]. It can directly predict the new state and action reward based on the model without interacting with the environment. In terms of update methods, MDP-Based RL can be divided into two categories by focusing on policy or value. The ValueBased RL outputs the action's value, and chooses the action with the highest value, while the Policy-Based RL outputs the probability of the next action, and selects actions based on the probability [13]. 
The optimal transport (OT) focuses on solving the optimal transport scheme between two different distributions, and its distance optimization problem is highly similar to the offloading problem that we need to solve, which is transferring tasks from EDs to computation nodes providing computing services [14]. We formulate joint optimization as an MDP problem and develop an Optimal-Transport-Based RL approach to solve it [15]. It is based on a Policy-Based RL to obtain a weighted sum that minimizes the processing delay of all tasks and end devices' energy consumption. Fusing the RL and the regularized annealing scheduling learning policy in the optimal transport can adaptively adjust the policy space during the RL exploration, thereby making the training process more stable.

The contributions of this paper are as follows:

- We propose a collaborative computation offloading model considering both delay and energy consumption, which can effectively utilize the computing resources among cloud servers and edge servers, and we formulate it as an optimization problem.

- To speed up the convergence, we introduce optimal transport into the exploration of Policy-Based RL and propose an Optimal-Transport-Based RL approach and construct a corresponding hybrid algorithm to solve the multi-objective optimization problem.

- We evaluate the performance of the proposed approach compared to other three baselines, and simulation results show that the proposed approach significantly reduces delay and energy consumption.

The remainder of this paper is organized as follows. The system model is presented in Section II. In Section III, we describe the problem formulation for computation offloading. In section IV, we propose the Optimal-Transport-Based RL approach to solve our proposed problem. The proposed approach's performance is evaluated and analyzed by simulation experiments in Section V. Finally, in Section VI, we conclude this paper.

\section{SySTEM MOdEL}

As illustrated in Fig. 1, we consider a wireless powered cloud-edge-end network, including EDs and computation nodes containing cloud servers and edge servers. In our computation offloading model, EDs can upload their tasks to the computation nodes for processing. Let $t_{i}=c_{i}, s_{i}$ represents the task from EDs, where $c_{i}$ represents the number of CPU cycles required to complete the task, and $s_{i}$ represents the input data size of the task. We use $d_{i}=-1,0,1$ to represent the offloading method of tasks, $d_{i}=-1$ means processing tasks on EDs, $d_{i}=0$ means that the task is offloaded by the edge base station to a nearby edge server for processing, and $d_{i}=1$ means that the task is offloaded to the cloud server for processing.

\section{A. Communication Model}

We assume that the channel state between EDs and base stations (BS) containing Small-Cell BS or Macro-Cell BS in the computation offloading model varies with time, that

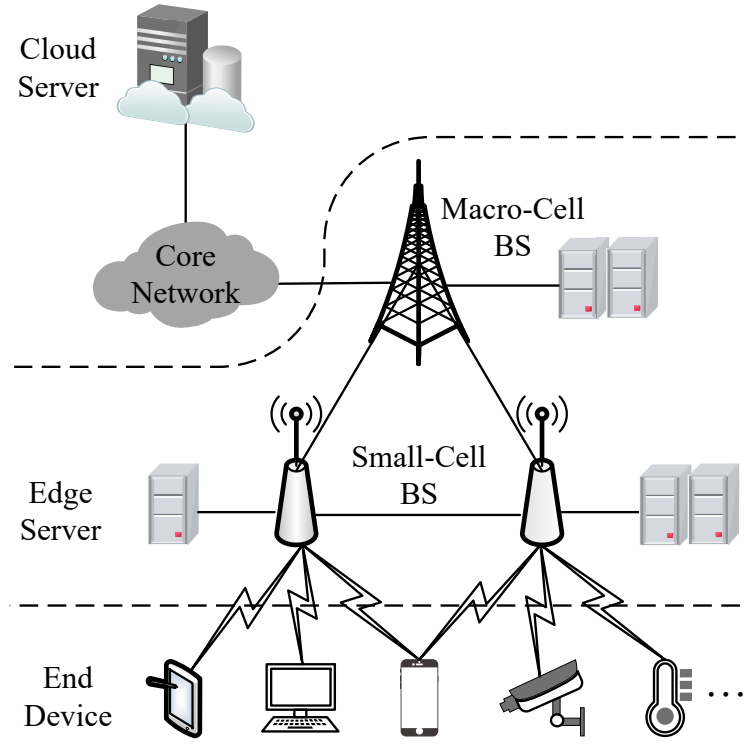

Fig. 1. Collaborative Computation Offloading Model.

is, the channel state is different in each time slot. There is only one eNB in one BS, so the interval interference is neglected. It's assumed that if multiple applications choose to offload the task simultaneously, the wireless bandwidth would be equally allocated to the offloading EDs for uploading data. If the user chooses the BS to execution task, the uplink communications are serving multiple users. Let $R^{\mu}$ denotes the channels' upload rate of the wireless network channel and can be obtained by:

$$
R^{\mu}=W \log _{2}\left(1+\frac{P^{\mu} G}{W V}\right),
$$

where $W$ is the wireless channel bandwidth, $P^{\mu}$ is the transmission power of the end device to upload data, $G$ is the Channel gain of the wireless channel allocated to the end device by the BS, and $V$ is the variance of the channel with Additive White Gaussian Noise.

\section{B. Delay and Energy Consumption Model}

In our work, different offloading methods correspond to different delays and energy consumption. Specifically, when EDs offloading tasks, the delay refers to the sum of the transmission time of offloading data to the computation node, the execution processing time at the computation node, and the transmission time of receiving the data result processed by the computation node, and the energy consumption refers to the sum of the transmission energy consumption of offloading data to the computation node and the transmission energy consumption of receiving the data result processed by the computation node.

a) Local Computing: When a task is offloaded for processing locally, we denote the local computing delay of task $t_{i}$ as $T_{i}^{\text {local }}$, and only relate to the computing capability $f^{e n d}$ 
and the required CPU-cycle frequency $c_{i}$. Therefore, the local computing delay can be obtained by:

$$
T_{i}^{l o c a l}=\frac{c_{i}}{f^{e n d}} .
$$

Let $P^{c}$ denotes the computing power, and the energy consumption for task is denoted by $E_{i}^{\text {local }}$ and is defined as:

$$
E_{i}^{\text {local }}=P^{c} \frac{c_{i}}{f^{e n d}} .
$$

b) Edge Computing: For the edge computing method, the EDs can offload the task to the edge server through wireless connection between EDs and Small-Cell BS. In addition, edge computing needs to upload the required data to the edge server. The execution time of task on the edge server is given by:

$$
T_{i}^{e d g e}=\frac{s_{i}}{R^{u}}+\frac{c_{i}}{f^{e d g e}},
$$

where $f^{e d g e}$ stands for the computing capability of the edge server. Let $P^{u}$ denotes the upload power, and $P^{i d l e}$ denotes the idle power. To sum up, the energy consumption can be obtained by:

$$
E_{i}^{e d g e}=P^{u} \frac{s_{i}}{R^{u}}+P^{i d l e} \frac{c_{i}}{f^{e d g e}} .
$$

c) Cloud Computing: In the case of offloading tasks to cloud servers, the processing delay can be divided into three aspects, including the transmission delay of the wireless channel, the transmission delay of the base station to the cloud servers, and the computational delay when the cloud server processes the task. Thus, the total cloud computing delay for task $T_{i}^{\text {cloud }}$ is as follows:

$$
T_{i}^{\text {cloud }}=\frac{s_{i}}{R^{u}}+\frac{s_{i}}{R^{\text {trans }}}+\frac{c_{i}}{f^{\text {cloud }}},
$$

where $f^{\text {cloud }}$ stands for the computing capability of the cloud server, and $R^{\text {trans }}$ is the transmission rate of the Small-Cell BS through the core network to cloud servers. To offload tasks to cloud servers, there is no processing energy consumption on EDs, but the transfer energy consumption of the data upload and the idle energy consumption of EDs need to be considered. Then, the total energy consumption of cloud computing can be calculated by:

$$
E_{i}^{\text {cloud }}=P^{u} \frac{s_{i}}{R^{u}}+P^{\text {idle }}\left(\frac{s_{i}}{R^{\text {trans }}}+\frac{c_{i}}{f^{\text {cloud }}}\right) .
$$

\section{PROBLEM FORMULATION}

Based on the system model in the above section, our objective is to minimize the overall cost of delay and energy consumption of all tasks by optimally choosing an offloading policy. We transform the original offloading problem into solving the optimization problem of the optimal transport scheme. We define $C(\alpha, \beta)$ as the objective cost function, where $\alpha=\sum_{i=1}^{N} a_{i} \delta_{x_{i}}$ denotes a discrete probability measurement of the task to be offloaded by the end device, and $\beta=\sum_{i=1}^{N} b_{i} \delta_{x_{i}}$ denotes another discrete probability measure for tasks that has already been processed [16]. According to the definition of Monge-Kantorovich transport problem, the optimization problem is formulated as follows:

$$
\begin{aligned}
& \min C(\alpha, \beta)=\min _{T} \int_{M} c(x, y) d \gamma(x, y) \\
& \text { s.t. }\left\{\begin{array}{l}
C 1: a=(1,0, \cdots 0) \in R^{N} \\
C 2: b=\left(0, b_{2}, \cdots, b_{N}\right) \in R^{N} \\
C 3: T_{\#} \alpha=\sum_{i=1}^{N} a_{i} \delta_{T^{-1}\left(x_{i}\right)}=\beta \\
C 4: \sum_{i=1}^{N} b_{i}=1
\end{array}\right.
\end{aligned}
$$

In (8), $C(\alpha, \beta)$ represents the transmission distance, i.e. the delay and energy consumption that comes with offloading. $\gamma(x, y)$ stands for the computing power of three different compute nodes. Constraints $\mathrm{C} 1$ and $\mathrm{C} 2$ define the vectors $\mathrm{a}$ and $\mathrm{b}$ that make up the discrete probability measurement $\alpha$ and $\beta$. Constraints $\mathrm{C} 3$ enforce that the tasks assigned to each compute node are consistent with the tasks waiting to be offloaded and the matrix transformation $T$ represents a manyto-many mapping of offloading decision, and $\mathrm{C} 4$ means that a task can only be processed by one compute node.

\section{Proposed SOLUTION}

In the above section, we formulate the optimization problem to optimize offloading performance. This section utilizes the Optimal-Transport-Based RL approach to design the corresponding algorithm and address the formulated optimization problem.

\section{A. Optimal Transport}

When faced with large-scale offloading, solving how to map multiple tasks from one space to another simultaneously, rather than just thinking about one task, is the sticking point to OT. Problem (8) is challenging to solve because constraint C3 is not linear, and Kantorovich Relaxation can relax the original constraint, allowing multiple tasks to be offloaded to multiple servers [14], [16]. Since the convex optimization problems are always paired, the convex maximization Problem (9) corresponding to the convex minimization Problem (8) is given as follows:

$$
\min C(\alpha, \beta)=\max _{f} \int_{M} f(x) d \alpha(x)+\int_{M} f^{c}(y) d \beta(y),
$$

and the corresponding meaning of Problem (9) is to maximize the total number of tasks that can be offloaded simultaneously by changing the overall cost of delay and energy consumption. Let $c(x, y)=d(x, y)=\|x-y\|_{2}$, then, Problem (10) is the dual problem of Problem (9) and can be solved by convex optimization method [17].

$$
\begin{aligned}
& \min C(\alpha, \beta)=\min _{s} \int_{M}\|s(x)\|_{2} d x, \\
& \text { s.t. } \nabla \cdot s(x)=\alpha(x)-\beta(x) .
\end{aligned}
$$




\section{B. RL for Computation Offloading}

OT is suitable for offline environments. For real-time systems that need to obtain real-time computing resources, offline offloading will bring an unbearable burden. RL methods can provide online updating and are widely used in offloading scheme, and the research emphasis of computation offloading based on RL is the learning of offloading policy. Specifically, first, observe its current state $s_{t}$, then take action $a_{t}$, and get its practical information, namely the immediate reward $r_{t}$ and new state $s_{t+1}$, will be used to adjust the offloading policy and this process will be repeated until the policy is close to the optimal policy. When the Policy-Based RL is used to approximate the offloading policy, it can be described as a policy function containing learning parameters $\theta[18]$.

$$
\pi_{\theta}(s, a)=P(a \mid s, \theta) \approx \pi(a \mid s)
$$

To maximize the long-term cumulative reward, the reward value $r_{i}$ can be defined as the cumulative reward after the current moment.

$$
r_{i}=R(s, a)=\gamma_{a} \frac{T^{\text {local }}-T^{o f f}}{T^{\text {local }}}+\gamma_{b} \frac{E^{\text {local }}-E^{o f f}}{E^{\text {local }}}
$$

where $T^{\text {local }}$ and $E^{\text {local }}$ indicate the delay and energy consumption of directly processing locally. $T^{o f f}$ and $E^{o f f}$ indicate the delay and energy consumption of offloading. $\gamma_{a}$ and $\gamma_{b}$ denotes the value range of weight factors which balance the delay and the energy consumption and avoid the infinite total reward when the time is too long. Furthermore, in order to evaluate the expected reward of the offloading policy, we define the expected reward obtained after performing an offloading action a under the state $\mathrm{s}$ as the State-Action Value function and is expressed as:

$$
Q_{\pi}(s, a)=\mathbb{E}_{\pi}\left[\sum_{k=0}^{\infty} \gamma^{k} r_{t+k} \mid S_{t}=s, A_{t}=a\right] .
$$

After expressing the offloading policy as a continuous function, the optimization methods of continuous function such as the gradient ascent can be used to find the optimal policy. This requires an objective function that can be optimized, which is represented by the average reward for each step as:

$$
J_{a v R}(\theta)=\sum_{s} d_{\pi_{\theta}}(s) \sum_{a} \pi_{\theta}(s, a) R(s, a),
$$

and the gradient of the derivative of (14) with respect to $\theta$ can be expressed as:

$$
\nabla_{\theta} L(\theta)=\mathbb{E} \pi_{\theta}\left[\nabla_{\theta} \log \pi_{\theta}(s, a) Q_{\pi}(s, a)\right] .
$$

Here, based on the Softmax function [19], the policy function $\pi_{\theta}(s, a)$ uses the linear combination of the characteristics describing the state $s$, action $a$ and the parameter $\theta$ to weigh the probability of next action is expressed as follows:

$$
\pi_{\theta}(s, a)=\frac{e^{\phi(s, a)^{T} \theta}}{\sum_{b} e^{\phi(s, b)^{T} \theta}} .
$$

\section{Optimal-Transport-Based RL}

In the above subsections, we use OT and RL to solve offline offloading and online updating, respectively. We combine OT and Policy-Based RL to propose an Optimal-Transport-Based RL (OTRL) approach, where OT is conducive to training a stable model, and RL explore the best offloading policy in the continuous learning process. The joint loss function $L_{O T R L}$ is written as:

$$
L_{O T R L}=\lambda_{1} C(\alpha, \beta)+\lambda_{2} J_{a v R}(\theta) .
$$

and the process can be divided into the following aspects:

- Offline training: The unique offloading decision obtained by solving cost function $C(\alpha, \beta)$ under given computing resources and tasks can train the offline model.

- RL exploration: RL acquires real-time computing and communication resources through continuous interaction and gradually expands the exploration space by introducing the Policy-Based RL objective function $J_{a v R}(\theta)$.

- OT fine-tune: We add cost function $C(\alpha, \beta)$ again into the iterative process of RL to fine-tune the model and accelerate the convergence of the model.

In addition, we can gradually increase or decrease $\lambda_{1}$ and $\lambda_{2}$ to achieve parameter annealing when training the offloading model. The OTRL algorithm for computation offloading is shown in Algorithm 1.

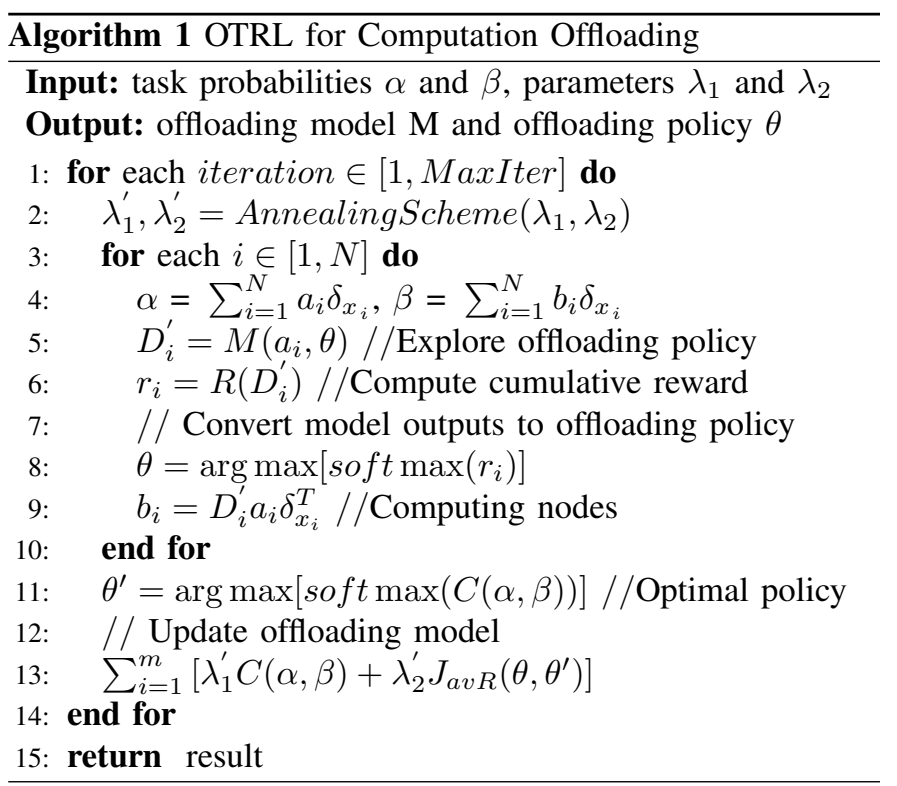

\section{Simulation ExPERIMENTS}

In this section, we verify the offloading performance of the Optimal-Transport-Based RL approach.

\section{A. Simulation Settings}

In simulation, we assume that the computation capacity of the end device, edge server, and cloud server are set to $1 \mathrm{GHz}, 10 \mathrm{GHz}$, and $100 \mathrm{GHz}$, respectively. The wireless channel bandwidth is $50 \mathrm{MHz}$, the number of channels is 50 , the number of the end device is 100 , and they are randomly 
distributed within the coverage of multiple base station. The wireless transmission power is $0.1 \mathrm{~W}$, the computation power of the end device is $0.5 \mathrm{~W}$, and the Additive White Gaussian Noise is $-100 \mathrm{dBm}$. The number of CPU cycles required by the edge server and cloud server are 200 cycles/bit and 50 cycles/bit. Moreover, we assume that the data size of tasks obeys a uniform distribution between [100,500]. On the basis of the above experimental parameter settings, we compare the proposed OTRL algorithm with the following three latest benchmark algorithms:

- DDPG algorithm: DDPG is a deep reinforcement learning technique adopted to achieve the optimal solution of the complicated nonlinear optimization problem [20].

- Greedy algorithm: For each task, a greedy strategy is used to decide to execute a task at its EDs or offload it to servers for processing to minimize the delay.

- Random algorithm: Without violating the original task dependencies, the performance of other offloading algorithms should be at least higher than this benchmark algorithm.

\section{B. Simulation Results and Analysis}

The experiment first verifies the convergence performance of the proposed OTRL algorithm in the offline training, and we take the loss function to highlight the convergence performance. As shown in Fig. 2, during the 50 rounds of training, it can be seen from the fitting curve that the value of loss function in two RL algorithms decreases rapidly. When the number of training reaches 30 , the joint loss function $L_{O T R L}$ tends to be stable. With the help of both OT and RL, it can be seen that the proposed OTRL algorithm converges fast and is more stable than DDPG algorithm.

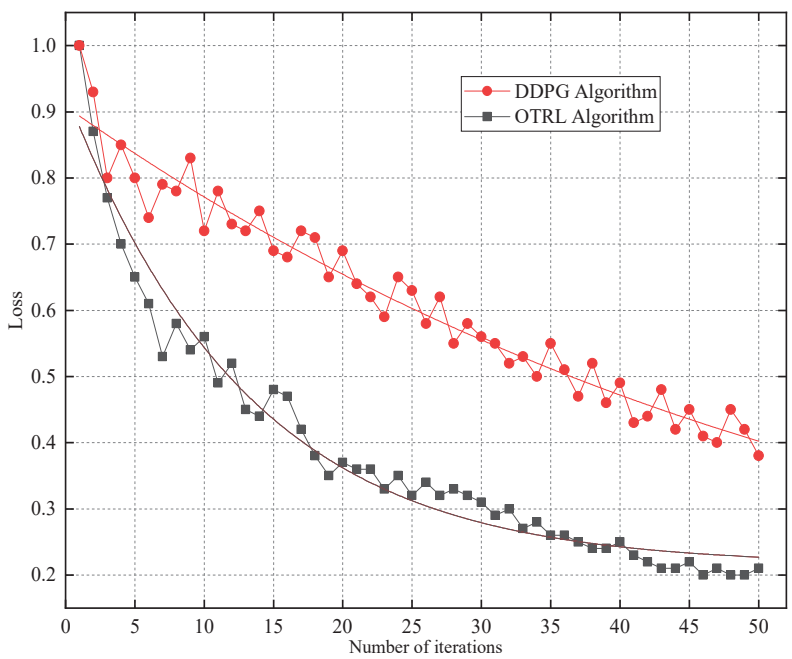

Fig. 2. Convergence curve of loss vs number of iterations.

Next, we consider how the the required data size during offloading impacts the offloading performance. Fig. 3 shows the average cost under scenarios where the required data ranges from $100 \mathrm{~KB}$ to $1000 \mathrm{~KB}$. In particular, the average cost of the Greedy algorithm and the Random algorithm increase relatively fast as the data size increases, while the average cost of the proposed OTRL algorithm increases relatively slowly. The figure shows that the proposed OTRL algorithm and the DDPG algorithm outperforms the other two benchmark algorithms because the increasing data leads to a more extensive search space for RL methods to obtain an optimal policy.

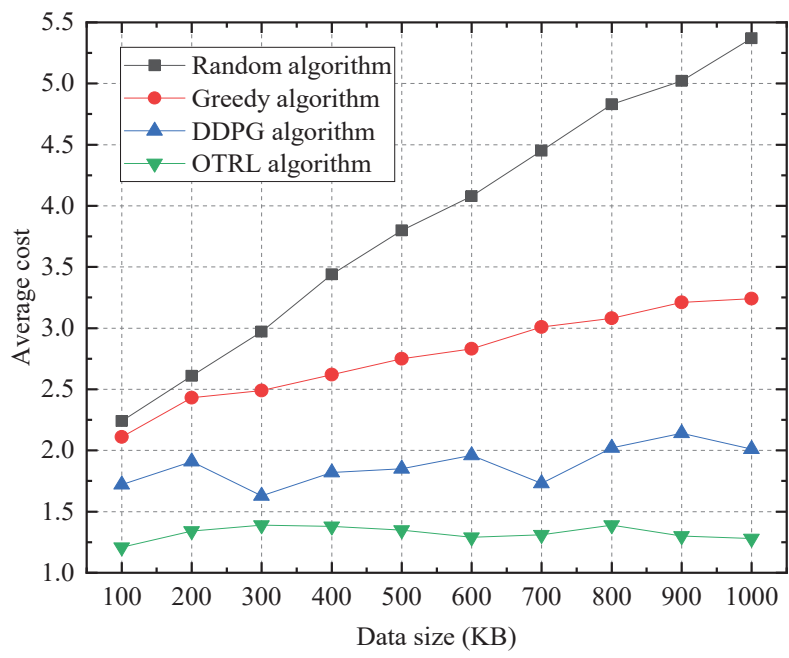

Fig. 3. Average cost under different data size.

As shown in Figs. 4, we consider the impact of different required $\mathrm{CPU}$ cycles to process the tasks, and the required CPU cycles for processing tasks ranges from 10 Gcycles to 20 Gcycles. Except for the Random algorithm and the Greedy algorithm, we can note that the average cost of the other two methods are relatively stable as the required CPU cycles change, which means they can adapt to different required resources dynamically. Furthermore, the proposed OTRL algorithm shows no clear sign of increasing. This is because the proposed algorithm can make intelligent offloading decisions to reduce the overall cost of delay and energy consumption.

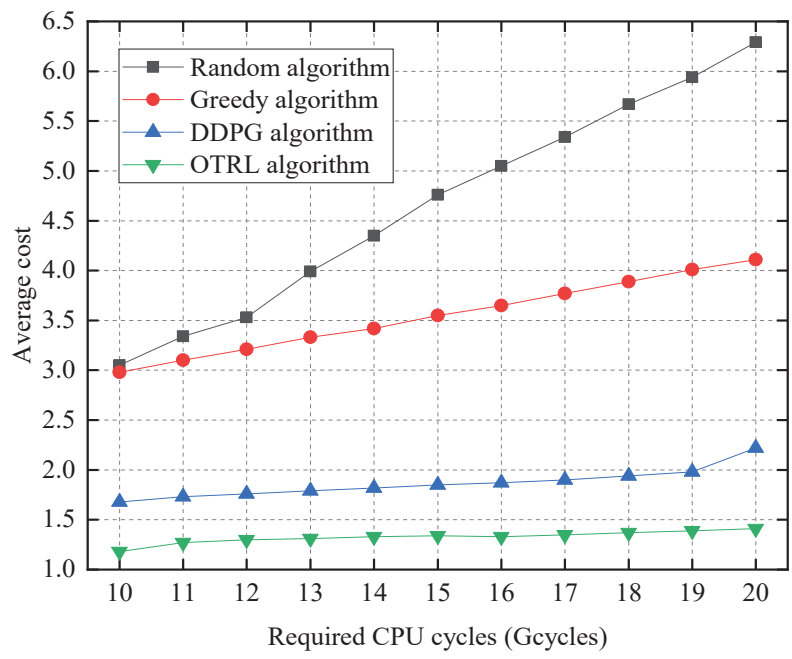

Fig. 4. Average cost under different required CPU cycles. 
In the previous two simulations, we obtained different average cost by changing task attributes containing data size and required CPU cycles. After standardized the input of the above two factors, we consider how the number of EDs impacts the offloading ratio. Fig. 5 shows the variation of offloading ratio as number of EDs change. We can observe that offloading ratios decreases with the increasing EDs and the OTRL algorithm outperforms the other three benchmark algorithms. What makes this phenomenon is that the OTRL algorithm can make full use of the collaborative resources of cloud servers and edge servers.

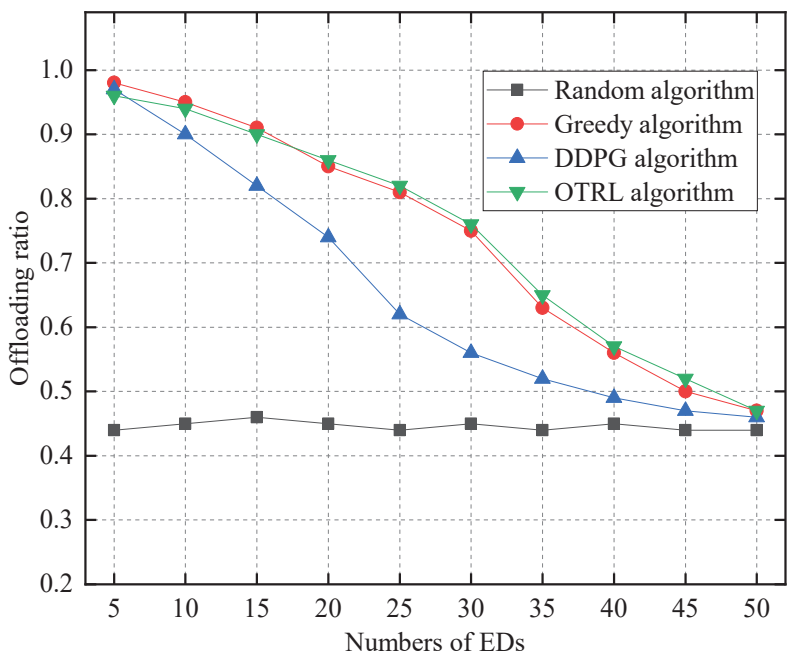

Fig. 5. Offloading ratio under different numbers of EDs.

\section{CONCLUSION}

In this paper, we have studied the problem of computation offloading in cloud and edge computing, where multiple EDs have computation-intensive and time-sensitive tasks that need to be executed either locally or remotely. Considering the situation that EDs can offload tasks to edge stations, and edge stations can further offload tasks to nearby edge server or cloud server. The problem is formulated as the joint optimization of delay and energy consumption. To solve the formulated multiobjective optimization problem, we have proposed an OptimalTransport-Based RL (OTRL) approach to find a solution for the intractable problem. Simulation results have demonstrated that the proposed approach can achieve a better trade-off performance on delay and energy consumption than other computation offloading schemes and decrease the complexity compared to the conventional RL methods.

\section{ACKNOWLEDGMENT}

This work was supported by the National Key R\&D Program of China (Grant No.2018YFB1800100), the National Natural Science Foundation of China (Grant No.U1909204), the Beijing Municipal Science and Technology Project (Grant No.Z191100007519007) and the Open Research Projects of Zhejiang Lab (Grant No.2021LC0AB03).

\section{REFERENCES}

[1] X. Chen, L. Jiao, W. Li and X. Fu, "Efficient Multi-User Computation Offloading for Mobile-Edge Cloud Computing," in IEEE/ACM Transactions on Networking, vol. 24, no. 5, pp. 2795-2808, October 2016.

[2] L. Qian, Y. Wu, F. Jiang, N. Yu, W. Lu and B. Lin, "NOMA assisted Multi-task Multi-access Mobile Edge Computing via Deep Reinforcement Learning for Industrial Internet of Things," in IEEE Transactions on Industrial Informatics, doi: 10.1109/TII.2020.3001355.

[3] W. Shi, J. Cao, Q. Zhang, Y. Li and L. Xu, "Edge Computing: Vision and Challenges," in IEEE Internet of Things Journal, vol. 3, no. 5, pp. 637-646, Oct. 2016.

[4] C. You, K. Huang, H. Chae and B. Kim, "Energy-Efficient Resource Allocation for Mobile-Edge Computation Offloading," in IEEE Transactions on Wireless Communications, vol. 16, no. 3, pp. 1397-1411, March 2017.

[5] P. Mach and Z. Becvar, "Mobile Edge Computing: A Survey on Architecture and Computation Offloading," in IEEE Communications Surveys \& Tutorials, vol. 19, no. 3, pp. 1628-1656, thirdquarter 2017.

[6] L. Yang , C. Zhong, Q. Yang, W. Zou and A. Fathalla, "Task offloading for directed acyclic graph applications based on edge computing in Industrial Internet," in Information Sciences, vol. 540, pp. 51-68, 2020.

[7] T. Q. Dinh, J. Tang, Q. D. La and T. Q. S. Quek, "Offloading in Mobile Edge Computing: Task Allocation and Computational Frequency Scaling," in IEEE Transactions on Communications, vol. 65, no. 8, pp. 3571-3584, Aug. 2017.

[8] X. Lin, Y. Wang, Q. Xie and M. Pedram, "Task Scheduling with Dynamic Voltage and Frequency Scaling for Energy Minimization in the Mobile Cloud Computing Environment," in IEEE Transactions on Services Computing, vol. 8, no. 2, pp. 175-186, 1 March-April 2015.

[9] M. B. Terefe, H. Lee, N. Heo, G. C. Fox, and S. Oh, "Energy-efficient multisite offloading policy using Markov decision process for mobile cloud computing," Pervasive and Mobile Computing, vol. 27, pp. 75-89, April 2016.

[10] J. Li, H. Gao, T. Lv and Y. Lu, "Deep reinforcement learning based computation offloading and resource allocation for MEC," 2018 IEEE Wireless Communications and Networking Conference (WCNC), Barcelona, pp. 1-6, 2018.

[11] X. Chen, H. Zhang, C. Wu, S. Mao, Y. Ji and M. Bennis, "Optimized Computation Offloading Performance in Virtual Edge Computing Systems Via Deep Reinforcement Learning," in IEEE Internet of Things Journal, vol. 6, no. 3, pp. 4005-4018, June 2019.

[12] J. Wang, J. Hu, G. Min, W. Zhan, Q. Ni and N. Georgalas, "Computation Offloading in Multi-Access Edge Computing Using a Deep Sequential Model Based on Reinforcement Learning," in IEEE Communications Magazine, vol. 57, no. 5, pp. 64-69, May 2019.

[13] C. Guestrin, R. Patrascu, D. Schuurmans, "Algorithm-directed exploration for model-based reinforcement learning in factored MDPs," in International Conference on Machine Learning, pp. 235-242, 2002.

[14] M. Cuturi, "Sinkhorn distances: Lightspeed computation of optimal transport," Advances in neural information processing systems, pp. 2292-2300, 2013.

[15] L. Chen , K Bai, C Tao, Y. Zhang and L Carin, "Sequence Generation with Optimal-Transport-Enhanced Reinforcement Learning," AAAI Conference on Artificial Intelligence, 2020.

[16] Y. Xie, X Wang, R. Wang and H. Zhang, "A fast proximal point method for Wasserstein distance," arXiv preprint arXiv:1802.04307. 2018.

[17] L. Condat, "A primal-dual splitting method for convex optimization involving Lipschitzian, proximable and linear composite terms," in Journal of Optimization Theory and Applications, vol. 158, no. 2, pp. 460-479, 2013.

[18] J. Wang, J. Hu, G. Min, W. Zhan, Q. Ni and N. Georgalas, "Computation Offloading in Multi-Access Edge Computing Using a Deep Sequential Model Based on Reinforcement Learning," in IEEE Communications Magazine, vol. 57, no. 5, pp. 64-69, May 2019.

[19] Y. Li, F. Qi, Z. Wang, X. Yu and S. Shao, "Distributed Edge Computing Offloading Algorithm Based on Deep Reinforcement Learning," in IEEE Access, vol. 8, pp. 85204-85215, 2020.

[20] C. Qiu, Y. Hu, Y. Chen and B. Zeng, "Deep Deterministic Policy Gradient (DDPG)-Based Energy Harvesting Wireless Communications," in IEEE Internet of Things Journal, vol. 6, no. 5, pp. 8577-8588, Oct. 2019. 